\title{
Evaluation of Medication Adherence and Associated Factors in Hypertensive Patients in Bangalore, India; A Cross-Sectional Study
}

\author{
Amar Prashad Chaudhary,"*, Jeswin George ${ }^{2}$, Kaveri Kadowade', Lubna Faiyza', Rabiya Anjum', Uma V M', Sharath K N \\ 'Department of Pharmacy Practice, Mallige College of Pharmacy, Bangalore, Karnataka, INDIA. \\ 2Department of Clinical Pharmacy, Mallige Hospital, Bangalore, Karnataka, INDIA.
}

\begin{abstract}
Background: This survey aims to determine medication compliance among hypertension patients and discover factors influencing medication compliance. Methods: This study incorporated a cross-sectional study design. The Hill-Bone Compliance to High Blood Pressure Therapy Scale was used in this study to evaluate medication adherence in hypertensive individuals. Additionally, this scale aids in monitoring sodium intake control and appointment-keeping habits. Results: 69 (77\%) of 90 hypertension patients comply with their antihypertensive therapy, whereas $21(23 \%)$ are non-compliant. The average score of patients on the Hill-Bone Compliance to High Blood Pressure Therapy Scale is $20.21 \pm 4.21$. Subsequently, the medication-taking score is $11.97 \pm 3.44$, the sodium reduction score is 4.37 \pm 1.4 , and the appointment-keeping score is $3.88 \pm 1.34$. Simultaneously, studying the impact of patients' socio-demographic characteristics and medical history on medication adherence. There was a considerable drop in the overall score of patients having more than five children by 5.59 $(P$-value $<.05)$, thus improving medication adherence. In contrast, the total score of patients with hypertension from 6 to 10 years shot up by
\end{abstract}

2.4 ( $P$-value $<.05)$, whereas the total score of patients with comorbidities more than 5 have a sharp rise of $3.4(P$-value $<.05)$. Therefore, reducing medication adherence. Conclusion: According to this survey, only $77 \%$ of patients are adherent to their antihypertensive medicines. Additionally, this study reveals that individuals with more than five children positively affect medication adherence. However, patients with long-term hypertension and many comorbidities have a detrimental effect.

Key words: Antihypertensive medication, Hill-Bone, Hypertension, Medical history, Medication Compliance.

\section{Correspondence}

Dr. Amar Prashad Chaudhary,

Department of Pharmacy Practice, Mallige College of Pharmacy, Rajiv Gandhi University of Health Sciences, Bangalore-560090, Karnataka, INDIA.

Email id: pamar419@gmail.com

DOI: 10.5530/ijpi.2021.4.76

\section{INTRODUCTION}

Almost one-fourth of the world's population is affected by hypertension and is estimated to affect 1.56 billion individuals by $2025 .{ }^{1,2}$ Hypertension is prevalent in $30.7 \%$ of the people in India and is significantly more frequent in those over the age of $65 .{ }^{3}$ Hypertension is asymptomatic for a lengthy period; hence, its detrimental effects may manifest years later. Uncontrolled hypertension surges the probability of developing cardiovascular disorders such as ischemic heart disease and can result in consequences such as stroke.

Despite the abundance of effective therapy, still, more than $50 \%$ of patients treated with hypertension quit within a year of diagnosis. Only about half of those take at least $80 \%$ of their prescription medicines. ${ }^{4}$ As a result of inadequate adherence to antihypertensive therapy, about two-thirds of individuals diagnosed with hypertension do not attain optimal blood pressure control. ${ }^{4}$ The situation in India isn't different, with about a quarter of the patient having poor medication adherence. ${ }^{5,6}$ Various factors have been associated with poor blood pressure controlone of the most crucial factors is non-adherence to medication. According to WHO, medication adherence is defined as "the extent to which a person's behaviour in taking medication, lifestyle management, diet, corresponding with agreed recommendations from a health care provider." 4 Some of the vital causes for poor compliance to the hypertensive medication in the Indian scenario was found to be the weak knowledge of hypertensive medicines among the patients, polypharmacy, multiple comorbidities, socioeconomic status, and educational status. ${ }^{5}$

Multiple studies have been conducted to study medication adherence among hypertensive patients in India. However, there is limited literature available to establish the influence of various factors on medication adherence. Therefore, the study aims to evaluate the medication adherence and factors affecting medication adherence among hypertensive patients.

\section{MATERIALS AND METHODS}

The research was carried out at Mallige Hospital in Bangalore, Karnataka's capital city. The cross-sectional study was conducted in December 2020 for five months. This study strictly adhered to Strengthening the Reporting of Observational Studies in Epidemiology (STROBE) standards for reporting. ${ }^{7}$

\section{Target population, setting, and sampling}

The target group consisted of adults (over the age of 18) who had been diagnosed with hypertension at least one year before data collection and were using at least one antihypertensive medication. This study did not exclude patients who had several comorbidities. This aided in determining the effect of additional comorbidities on the participant's medication compliance level. In addition, this study excluded individuals who had been diagnosed with hypertension but were younger than 18 years old, had been diagnosed with hypertension for less than one year or were not taking any antihypertensive medication. The patient's blood pressure was checked using a sphygmomanometer and categorized into control and uncontrolled blood pressure

This study recruited a convenience sample of 98 people, of which 8 were removed due to missing data, failing to meet the criteria, or providing 
misleading information. Data were collected from inpatient departments apart from the critical care unit (ICU) and surgery unit for two months. In those two months, an estimated 150 hypertension patients were hospitalized. The sample size for this study was determined using the convenience sampling method; the sample size is 90 for this study. ${ }^{8}$

\section{Instrument}

The data collection instrument was made up of three pieces. The first section included a questionnaire on the participants' socio-demographics. The second section had the participants' medical history. The third section included a scale to assess patient medication compliance. The Hill-Bone Compliance to High Blood Pressure Therapy Scale (Hill-Bone CHBPTS), developed by Kim et al., is used in the third section to assess patient compliance with antihypertensive medication therapy. ${ }^{9}$ This scale satisfies the study's objectives and has been cross-culturally validated. ${ }^{9-11}$ The Hill-Bone CHBPTS questionnaire consists of 14 items divided into three unique subscales. Subscales include a scale for reducing sodium intake, keeping appointments and medication use. ${ }^{9}$ The questionnaire responses were coded on a four-point Likert scale. None of the time equals 1 , part of the time equals 2 , most of the time equals 3 , and all of the time equals 4 . The overall score goes from 14 to 56 . The higher the patient's drug adherence, the lower the total score.

\section{Operational definition}

\section{Medication Compliance}

Patients who comply at a rate of $80 \%$ or more are called compliant, whereas those who comply at less than $80 \%$ are labelled non-compliant. ${ }^{6,12}$ The formula calculates the percentage of compliance

$$
\% \text { compliant }=\frac{56-\text { Total score }}{42}{ }^{2} 100 \%
$$

\section{Polypharmacy}

The regular use of five or more drugs is termed Polypharmacy. ${ }^{13}$

\section{Blood Pressure}

Uncontrolled blood pressure is defined as blood pressure equal to or greater than $140 / 90 \mathrm{~mm} \mathrm{Hg}$, while controlled blood pressure is defined as less than $140 / 90 \mathrm{~mm} \mathrm{Hg}{ }^{14}$

\section{Statistical analysis}

All the responses from the patient were recorded in Microsoft excel and were checked for the accuracy of the data. Statistical Package for Social Science (SPSS) version 25 software were used for the statistical analysis. The data analysis procedure included descriptive statistics to describe the sample. For example, the mean and standard deviation was used to describe continuous variables whereas, frequency and percentage were used to describe categorical variables. In addition, linear regression was used to understand the impact of medical history and sociodemographics of the patient on the Hill-Bone CHBPTS.

\section{Ethical Consideration}

The Mallige College of Pharmacy's research review board accepted our study (Approval no. MCP/RRB/004/19-20). The Helsinki Declaration of 1964 and its recent amendments were strictly followed in this study. ${ }^{15}$ The patients were explained the purpose of this study prior to the interview, and their verbal consent was taken.

\section{RESULTS}

Table 1 summarises the socio-demographic features of the patients who contributed to this investigation. Of the 90 patients, 51 (56.7\%) were male, and 39 (43.3\%) were female. Among the 90 patients, the majority (46\%) of them belonged to age group of 66 to 85 years, while 32 (35.6\%) were between the ages of 51 and $65,8(8.9 \%)$ were in the age range of 35 to 50 , and $6(6.7 \%)$ were in the age group of $>85$. While 38 patients $(42.2 \%)$ have a university education, 27 patients (30\%) have a high school education, and 10 patients (11.1\%) have only an elementary school education. Simultaneously, 15 patients (16.7\%) lacked literacy. Of the 90 patients, 37 (41.1\%) were normal weight, 31 (34.4\%) were overweight, $16(17.8 \%)$ were obese, and $6(6.7 \%)$ were underweight. Most (95.4\%) of the patients were married; however, 5 (5.6\%) were widows. Only 10 were smokers $(11.1 \%)$ out of 90 patients. The majority of patients (54.4\%) have between one and two children, whereas 36 patients $(40 \%)$ have between 3 and 5 children, and 5 patients (5.6\%) have more than 5 children.

The medical history of the patient is summarised in Table 2. Among 90 patients, almost one-third of patients had uncontrolled blood pressure. Of 90 patients, 36 (40\%) had hypertension for one to five years, $22(24.4 \%)$ had hypertension for six to ten years, and 32 (35.6\%) had hypertension for more than ten years. The patients were on $1.5 \pm .78$ of antihypertensive medicine and took $1.63 \pm .97$ daily antihypertensive pills. This study discovered that patients took an average of $4.6 \pm 3.7$ medications, and more than one-third were on polypharmacy. In addition, 15 patients

\section{Table 1: Socio-demographics of the patient.}

\begin{tabular}{|c|c|}
\hline Socio-demographic Details & Frequency $(\%)(n=90)$ \\
\hline \multicolumn{2}{|l|}{ Gender } \\
\hline Male & $51(56.7 \%)$ \\
\hline Female & $39(43.3 \%)$ \\
\hline \multicolumn{2}{|l|}{ Age (in years) } \\
\hline $35-50$ & $8(8.9 \%)$ \\
\hline $51-65$ & $32(35.6 \%)$ \\
\hline $66-85$ & $44(48.9 \%)$ \\
\hline$>85$ & $6(6.7 \%)$ \\
\hline \multicolumn{2}{|l|}{ Body Mass Index (BMI) } \\
\hline Underweight & $6(6.7 \%)$ \\
\hline Normal & $37(41.1 \%)$ \\
\hline Overweight & $31(34.4 \%)$ \\
\hline Obese & $16(17.8 \%)$ \\
\hline \multicolumn{2}{|l|}{ Education } \\
\hline Illiterate & $15(16.7 \%)$ \\
\hline Primary School & $10(11.1 \%)$ \\
\hline High School & $27(30 \%)$ \\
\hline University & $38(42.2 \%)$ \\
\hline \multicolumn{2}{|l|}{ Marital Status } \\
\hline Married & $85(95.4 \%)$ \\
\hline Widow & $5(5.6 \%)$ \\
\hline \multicolumn{2}{|l|}{ No. of children } \\
\hline $1-2$ & $49(54.4 \%)$ \\
\hline $3-5$ & $36(40 \%)$ \\
\hline$>5$ & $5(5.6 \%)$ \\
\hline \multicolumn{2}{|l|}{ Smoking Status } \\
\hline Yes & $10(11.1 \%)$ \\
\hline No & $80(88.9 \%)$ \\
\hline
\end{tabular}


Table 2: Medical history of the patients.

\begin{tabular}{|c|c|}
\hline $\begin{array}{c}\text { Medical History } \\
\text { Blood Pressure }(n=90)\end{array}$ & Distribution \\
\hline $\begin{array}{c}\text { Controlled } \\
\text { Uncontrolled }\end{array}$ & $62(68.9 \%)$ \\
\hline Duration of Hypertension (in years) $(n=90)$ & $28(31.1 \%)$ \\
\hline $1-5$ & $36(40 \%)$ \\
\hline $6-10$ & $22(24.4 \%)$ \\
\hline Total number of antihypertensive drugs prescribed \\
(mean \pm SD) & $32(35.6 \%)$ \\
\hline $\begin{array}{c}\text { Total number of drugs prescribed (mean } \pm \text { SD) } \\
\text { Total number of antihypertensive pills per day } \\
(\text { mean } \pm \text { SD) }\end{array}$ & $1.5 \pm .78$ \\
\hline No. of Patient on Polypharmacy $(n=90)$ & $4.6 \pm 3.7$ \\
\hline Total number of other comorbidities & $38(42.2 \%)$ \\
\hline 0 & $15(16.7 \%)$ \\
\hline 1 & $26(28.9 \%)$ \\
\hline 2 & $24(26.7 \%)$ \\
\hline 3 & $10(11.1 \%)$ \\
\hline$\geq 4$ & $15(16.7 \%)$ \\
\hline
\end{tabular}

Table 3: Domains of the Hill-Bone CHPTS.

\begin{tabular}{|c|c|c|c|c|c|c|}
\hline & 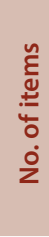 & $\begin{array}{l}\varepsilon \\
\frac{\varepsilon}{\xi} \\
\frac{E}{\Sigma} \\
\Sigma\end{array}$ & $\begin{array}{l}\xi \\
\underline{\underline{z}} \\
\frac{\xi}{x} \\
\frac{\pi}{\Sigma}\end{array}$ & 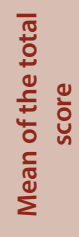 & 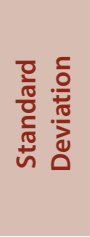 & 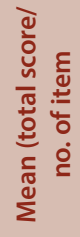 \\
\hline Reducing sodium intake & 3 & 3 & 9 & 4.37 & 1.4 & 1.45 \\
\hline Appointment keeping & 2 & 2 & 8 & 3.88 & 1.34 & 1.94 \\
\hline Medication taking & 9 & 9 & 28 & 11.97 & 3.44 & 1.33 \\
\hline Total Score & 14 & 14 & 37 & 20.21 & 4.21 & 1.44 \\
\hline
\end{tabular}

(16.7\%) had no comorbidity other than hypertension, 26 patients (28.9\%) had one, 24 patients $(26.7 \%)$ had two, 10 patients $(11.1 \%)$ had three, and 15 patients (16.7\%) had four or more comorbidities.

Table 3 summarises the Domains of the Hill-Bone CHPTS. The findings of this study indicated that overall adherence to hypertension treatment regimens was moderate to good, with a score of 1.44 , where 1 shows the highest potential rate of compliance and 4 marks the lowest. Adherence to sodium restriction and medication use was moderate to good, with a score of 1.45 and 1.33, respectively. Appointment punctuality was moderate among patients, with a score of 1.94. The patient's compliance with the antihypertensive treatment is illustrated in Figure 1. According to this study, 69 (77\%) patients comply with their antihypertensive medication therapy, whereas 21 patients (23\%) are non-compliant.

Table 4 summarises the effect of socio-demographic variables on the HillBone CHBPTS scale and its subscale. Among the identified risk factors, the Hill-Bone CHBPTS scale and its subscale are significantly affected by the number of children. It is discovered that when the number of children increases, medication compliance improves significantly. When a patient has more than five children, their total score decreases by 5.59

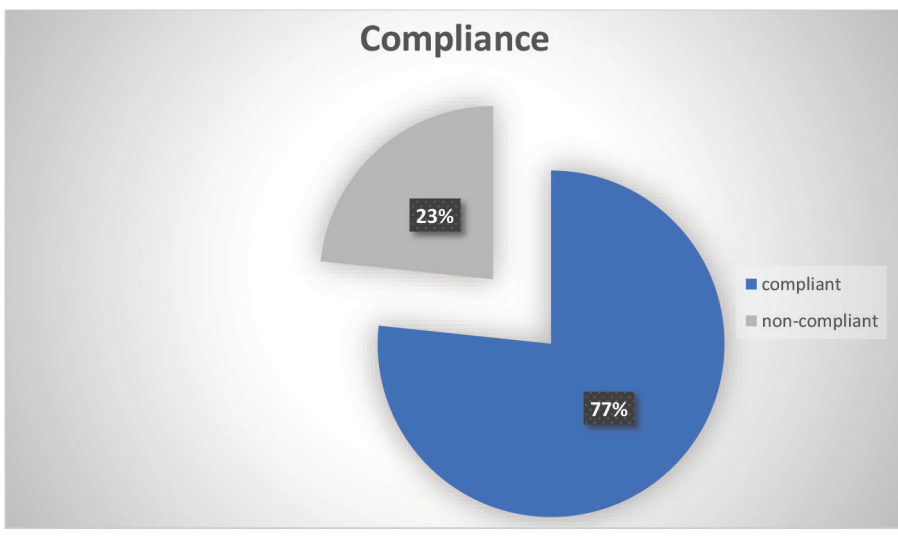

Figure 1: Compliance of patients with antihypertensive medications.

$(P$-value $<.05)$, while their medication-taking subscale score decreases by 4.94 ( $P$-value $<.05)$. Furthermore, the patient has a number of children between 3 to 5 ; their sodium intake subscale score was reduced by $.789(P$-value $<.05)$.

The effect of a patient's medical history on the Hill-Bone CHBPTS and its subscale scores is summarised in Table 5. Among the variables discovered, the duration of hypertension and the number of comorbidities other than hypertension have a substantial adverse effect on the Hill-Bone CHBPTS and its subscale. According to this study, patient's total score increases by $2.4(P$-value $<.05)$, and medication-taking scale score increases by $2.1(P$-value $<.05)$ when they have hypertension for 6 to 10 years. Furthermore, when patients have four or more comorbidities, their total score and medication-taking subscale score increase by 3.4 and 2.8 points, respectively $(P$-value $<.05)$. As a result, the patient's medication compliance and medication-taking behaviour deteriorate.

\section{DISCUSSION}

The primary goal of this study is to ascertain patient compliance with antihypertensive treatment. Additionally, this study reveals the variables that influence drug adherence in hypertension patients. According to this study, $77 \%$ of those individuals adhere to their antihypertensive medications, whereas $23 \%$ aren't compliant. Furthermore, this study discovered that as the number of children increases, medication compliance improves significantly. In contrast, a patient with four or more comorbidities other than hypertension and who has had hypertension for six to ten years reduces medication compliance.

The average Hill-Bone CHBPTS score for the patients in this research was $20.21 \pm 4.21$, which is comparable with the findings of a national survey performed by Chia et al. in Malaysia. ${ }^{2}$ When comparing the subscales, Malaysians had better medication adherence than the participants in our study but somewhat worse appointment keeping and salt intake control. ${ }^{2}$ It is probably because Malaysians have higher literacy, per capita income and the government's public health expenditure is $3.8 \%$ of its GDP. On the other hand, India has low literacy, per capita income, and the government's public health expenditure is estimated to be $1.28 \%$ of its GDP. Similarly, the study conducted in Poland by Uchmanowicz et al. was consistent with the findings of this study. ${ }^{16}$

Furthermore, a survey done in a tertiary care hospital in India by E. Mutneja et al. discovered that $80.8 \%$ of patients adhere to their antihypertensive therapy, similar to this study's findings. ${ }^{6}$ Compared to Palestinians residing in the Gaza Strip, the patients in this research have a higher rate of medication adherence and salt control but a lower rate of appointment keeping. ${ }^{17}$ The plausible reason is due to higher poverty and inadequate healthcare facilities among the Palestinian people. 
Table 4: Impact of socio-demographic factors on the Hill-Bone CHBPTS and its subscale score.

\begin{tabular}{|c|c|c|c|c|c|c|c|c|}
\hline \multirow{3}{*}{$\begin{array}{l}\text { Socio-demographic } \\
\text { characteristics }\end{array}$} & \multirow{2}{*}{\multicolumn{2}{|c|}{$\begin{array}{c}\text { Hill-Bone CHBPTS } \\
\text { Total Score }\end{array}$}} & \multicolumn{6}{|c|}{ Hill-Bone CHBPTS Sub-Scale } \\
\hline & & & \multicolumn{2}{|c|}{ Medication-Taking } & \multicolumn{2}{|c|}{ Appointment keeping } & \multicolumn{2}{|c|}{ Reducing Sodium intake } \\
\hline & $P$-value & $\begin{array}{c}\text { B } \\
(95 \% \mathrm{Cl})\end{array}$ & $P$-value & $\begin{array}{c}\text { B } \\
(95 \% \mathrm{Cl})\end{array}$ & $P$-value & $\begin{array}{c}\text { B } \\
(95 \% \mathrm{Cl})\end{array}$ & $P$-value & $\begin{array}{c}\text { B } \\
(95 \% \mathrm{Cl})\end{array}$ \\
\hline Constant & 0.000 & $\begin{array}{c}21.41 \\
(16.4-26.3)\end{array}$ & 0.000 & $\begin{array}{c}11.245 \\
(7.2-15.2)\end{array}$ & 0.000 & $\begin{array}{c}4.518 \\
(2.8-6.1)\end{array}$ & 0.000 & $\begin{array}{c}5.647 \\
(4-7.2)\end{array}$ \\
\hline Gender $^{\mathrm{a}}$ & 0.549 & $\begin{array}{c}-.7 \\
(-3-1.6)\end{array}$ & 0.528 & $\begin{array}{c}-.592 \\
(-2.4-1.2)\end{array}$ & 0.918 & $\begin{array}{c}-.04 \\
(-.8-.7)\end{array}$ & 0.857 & $\begin{array}{c}-.068 \\
(-0.8-0.6)\end{array}$ \\
\hline \multicolumn{9}{|l|}{ Age (in years) ${ }^{\mathrm{b}}$} \\
\hline $36-50$ & 0.786 & $\begin{array}{c}-.49 \\
(-4.1-3.1)\end{array}$ & 0.510 & $\begin{array}{c}-.973 \\
(-3.9-1.9)\end{array}$ & 0.955 & $\begin{array}{c}.035 \\
(-1.1-1.2)\end{array}$ & 0.460 & $\begin{array}{c}.44 \\
(-0.7-1.6)\end{array}$ \\
\hline $51-66$ & 0.489 & $\begin{array}{c}-.723 \\
(-1.3-2.7)\end{array}$ & 0.484 & $\begin{array}{c}.588 \\
(-1-2.2)\end{array}$ & 0.703 & $\begin{array}{c}-.134 \\
(-0.8-0.5)\end{array}$ & 0.428 & $\begin{array}{c}.268 \\
(-0.4-0.9)\end{array}$ \\
\hline$>85$ & 0.504 & $\begin{array}{c}1.6 \\
(-3.1-6.3)\end{array}$ & 0.359 & $\begin{array}{c}1.772 \\
(-2-5.5)\end{array}$ & 0.965 & $\begin{array}{c}.035 \\
(-1.5-1.6)\end{array}$ & 0.795 & $\begin{array}{c}-.201 \\
(-1.7-1.3)\end{array}$ \\
\hline \multicolumn{9}{|l|}{$\begin{array}{c}\text { (Body Mass Index) } \\
\text { BMI }^{\mathbf{c}}\end{array}$} \\
\hline Underweight & 0.592 & $\begin{array}{c}-1.22 \\
(-5.7-3.3)\end{array}$ & 0.628 & $\begin{array}{c}-.891 \\
(-4.5-2.7)\end{array}$ & 0.723 & $\begin{array}{c}-.272 \\
(-1.7-1.2)\end{array}$ & 0.930 & $\begin{array}{c}-.065 \\
(-1.5-1.4)\end{array}$ \\
\hline Overweight & 0.44 & $\begin{array}{c}.896 \\
(-1.4-3.1)\end{array}$ & 0.490 & $\begin{array}{c}.643 \\
(-1.2-2.4)\end{array}$ & 0.799 & $\begin{array}{c}.099 \\
(-0.6-0.8)\end{array}$ & 0.680 & $\begin{array}{c}.154 \\
(-.5-0.9)\end{array}$ \\
\hline Obese & 0.858 & $\begin{array}{c}-.26 \\
(-3.1-2.6)\end{array}$ & 0.904 & $\begin{array}{c}-.142 \\
(-2.4-2.1)\end{array}$ & 0.726 & $\begin{array}{c}-.172 \\
(-1.1-0.8)\end{array}$ & 0.912 & $\begin{array}{c}.052 \\
(-.8-0.9)\end{array}$ \\
\hline \multicolumn{9}{|l|}{ Education $^{\mathrm{d}}$} \\
\hline Illiterate & 0.443 & $\begin{array}{c}1.24 \\
(-1.9-4.4)\end{array}$ & 0.235 & $\begin{array}{c}1.553 \\
(-1 .-4.1)\end{array}$ & 0.953 & $\begin{array}{c}-.032 \\
(-1.1-1)\end{array}$ & 0.599 & $\begin{array}{c}-.276 \\
(-1.3-0.7)\end{array}$ \\
\hline Primary School & 0.563 & $\begin{array}{c}.951 \\
(-2.3-4.2)\end{array}$ & 0.127 & $\begin{array}{c}2.025 \\
(-.5-4.6)\end{array}$ & 0.607 & $\begin{array}{c}-.283 \\
(-1.3-0.8)\end{array}$ & 0.139 & $\begin{array}{c}-.791 \\
(-1.8-0.2)\end{array}$ \\
\hline High School & 0.616 & $\begin{array}{c}-.642 \\
(-3.1-1.8)\end{array}$ & 0.649 & $\begin{array}{c}-.468 \\
(-2.5-1.5)\end{array}$ & 0.694 & $\begin{array}{c}-.169 \\
(-1-.6)\end{array}$ & 0.990 & $\begin{array}{c}-.005 \\
(-.8-.8)\end{array}$ \\
\hline Marital Status $^{e}$ & 0.872 & $\begin{array}{c}-.373 \\
(-4.9-4.2)\end{array}$ & 0.557 & $\begin{array}{c}1.096 \\
(-2.6-4.7)\end{array}$ & 0.670 & $\begin{array}{c}-.331 \\
(-1.8-1.2)\end{array}$ & 0.132 & $\begin{array}{c}-1.138 \\
(-2.6-.3)\end{array}$ \\
\hline \multicolumn{9}{|l|}{ No. of Children ${ }^{f}$} \\
\hline $3-5$ & 0.085 & $\begin{array}{c}-1.84 \\
(-3.9-0.2)\end{array}$ & 0.386 & $\begin{array}{c}-.743 \\
(-2.4-.9)\end{array}$ & 0.376 & $\begin{array}{c}-.317 \\
(-1-0.3)\end{array}$ & 0.024 & $\begin{array}{c}-.789 \\
(-1.4--0.1)\end{array}$ \\
\hline$>5$ & $0.028^{*}$ & $\begin{array}{c}-5.59 \\
(-10.5--0.6)\end{array}$ & $0.016^{*}$ & $\begin{array}{c}-4.943 \\
(-8.9--.9)\end{array}$ & 0.418 & $\begin{array}{c}-.682 \\
(-2.3-0.9)\end{array}$ & 0.973 & $\begin{array}{c}.027 \\
(-1.5-1.6)\end{array}$ \\
\hline Smoking Status ${ }^{\mathrm{g}}$ & 0.915 & $\begin{array}{c}-.17 \\
(-3.4-3)\end{array}$ & 0.388 & $\begin{array}{c}-1.144 \\
(-3.7-1.4)\end{array}$ & 0.945 & $\begin{array}{c}.038 \\
(-1-1.1)\end{array}$ & 0.084 & $\begin{array}{c}.930 \\
(-.1-1.9)\end{array}$ \\
\hline $\mathrm{R}$ square & \multicolumn{2}{|c|}{0.122} & \multicolumn{2}{|c|}{.155 } & \multicolumn{2}{|c|}{.037} & \multicolumn{2}{|c|}{.180} \\
\hline
\end{tabular}

Multiple linear regression is statistically significant at the $P$-value $<0.05$ level

Reference category

${ }^{\mathrm{a}}$ Gender: Female

${ }^{\mathrm{b}}$ Age (in years): $66-85$

'BMI: Normal

dEducation: University

${ }^{\mathrm{e}}$ Marital status: widow

fNumber of children: $1-2$

${ }^{\mathrm{g} S}$ moking status: non smokers 
Table 5: Impact of the medical history of patients on Hill-Bone CHBPTS and its subscale.

\begin{tabular}{|c|c|c|c|c|c|c|c|c|}
\hline \multirow[t]{3}{*}{ Medical History } & \multirow{2}{*}{\multicolumn{2}{|c|}{$\begin{array}{c}\text { Hill-Bone CHBPTS } \\
\text { Total Score }\end{array}$}} & \multicolumn{6}{|c|}{ Hill-Bone CHBPTS Sub-Scale } \\
\hline & & & \multicolumn{2}{|c|}{ Medication Taking } & \multicolumn{2}{|c|}{ Appointment keeping } & \multicolumn{2}{|c|}{ Reducing Sodium intake } \\
\hline & $P$-value & $\begin{array}{c}\text { B } \\
(95 \% \mathrm{Cl})\end{array}$ & $P$-value & $\begin{array}{c}\text { B } \\
(95 \% \mathrm{Cl})\end{array}$ & $P$-value & $\begin{array}{c}\text { B } \\
(95 \% \mathrm{Cl})\end{array}$ & $P$-value & $\begin{array}{c}\text { B } \\
(95 \% \mathrm{Cl})\end{array}$ \\
\hline Constant & 0.000 & $\begin{array}{c}17.9 \\
(15.2-20.5)\end{array}$ & 0.000 & $\begin{array}{c}10.3 \\
(8.1-12.4)\end{array}$ & 0.000 & $\begin{array}{c}3.6 \\
(2.8-4.5)\end{array}$ & 0.000 & $\begin{array}{c}3.9 \\
(3-4.8)\end{array}$ \\
\hline Blood Pressure $^{a}$ & 0.608 & $\begin{array}{c}-0.52 \\
(-2.5-1.4)\end{array}$ & 0.917 & $\begin{array}{c}.087 \\
(-1.5-1.7)\end{array}$ & 0.523 & $\begin{array}{c}-.2 \\
(-0.8-0.4)\end{array}$ & 0.266 & $\begin{array}{c}-.39 \\
(-1-0.3)\end{array}$ \\
\hline \multicolumn{9}{|l|}{ Duration of hypertension ${ }^{\mathrm{b}}$} \\
\hline $6-10$ years & $0.036^{*}$ & $\begin{array}{c}2.4 \\
(0.1-4.7)\end{array}$ & $0.029^{*}$ & $\begin{array}{c}2.1 \\
(0.2-3.9)\end{array}$ & 0.706 & $\begin{array}{c}-0.14 \\
(-0.9-0.6)\end{array}$ & 0.202 & $\begin{array}{c}0.51 \\
(-0.2-1.3)\end{array}$ \\
\hline$>10$ years & 0.410 & $\begin{array}{c}0.88 \\
(-1.2-3)\end{array}$ & 0.286 & $\begin{array}{c}.94 \\
(-0.8-2.6)\end{array}$ & .576 & $\begin{array}{c}0.202 \\
(-0.5-0.9)\end{array}$ & 0.496 & $\begin{array}{c}-.253 \\
(-0.9-0.4)\end{array}$ \\
\hline $\begin{array}{l}\text { Total number of drugs } \\
\text { prescribed }\end{array}$ & 0.279 & $\begin{array}{c}0.22 \\
(-.1-.6)\end{array}$ & 0.330 & $\begin{array}{c}.16 \\
(-0.1-0.5)\end{array}$ & .091 & $\begin{array}{c}.11 \\
(-0.01-0.2)\end{array}$ & 0.413 & $\begin{array}{c}-.059 \\
(-0.2-0.08)\end{array}$ \\
\hline $\begin{array}{c}\text { Total number of } \\
\text { antihypertensive drugs } \\
\text { prescribed }\end{array}$ & 0.653 & $\begin{array}{c}-0.3 \\
(-1.6-1)\end{array}$ & 0.563 & $\begin{array}{c}-.32 \\
(-1.4-0.7)\end{array}$ & .974 & $\begin{array}{c}.007 \\
(-0.4-0.4)\end{array}$ & 0.974 & $\begin{array}{c}0.008 \\
(-0.4-0.4)\end{array}$ \\
\hline $\begin{array}{c}\text { Total number of } \\
\text { antihypertensive pills intake } \\
\text { per day }\end{array}$ & 0.558 & $\begin{array}{c}-0.35 \\
(-.8-1.5)\end{array}$ & 0.605 & $\begin{array}{c}.25 \\
(-.7-1.2)\end{array}$ & 0.630 & $\begin{array}{c}-.097 \\
(-.4-0.3)\end{array}$ & 0.348 & $\begin{array}{c}.19 \\
(-.2-0.6)\end{array}$ \\
\hline Polypharmacy ${ }^{c}$ & 0.256 & $\begin{array}{c}-1.8 \\
(-4.9-1.3)\end{array}$ & 0.189 & $\begin{array}{c}-1.7 \\
(-4.2-0.8)\end{array}$ & 0.325 & $\begin{array}{c}-.52 \\
(-1.5-0.5)\end{array}$ & 0.438 & $\begin{array}{c}.42 \\
(-.6-1.5)\end{array}$ \\
\hline \multicolumn{9}{|l|}{ No. of other comorbidities ${ }^{d}$} \\
\hline No comorbidity & 0.121 & $\begin{array}{c}2.1 \\
(-.5-4.8)\end{array}$ & 0.179 & $\begin{array}{c}1.5 \\
(-.7-3.7)\end{array}$ & .720 & $\begin{array}{c}.165 \\
(-0.7-1.0)\end{array}$ & 0.328 & $\begin{array}{c}.46 \\
(-0.4-1.4)\end{array}$ \\
\hline 2 comorbidities & 0.440 & $\begin{array}{c}.92 \\
(-1.4-3.2)\end{array}$ & 0.488 & $\begin{array}{c}.67 \\
(-1.2-2.6)\end{array}$ & .991 & $\begin{array}{c}-.004 \\
(-0.7-0.7)\end{array}$ & 0.543 & $\begin{array}{c}.25 \\
(-0.5-1)\end{array}$ \\
\hline 3 comorbidities & 0.904 & $\begin{array}{c}-.19 \\
(-3.3-2.9)\end{array}$ & 0.581 & $\begin{array}{c}-.72 \\
(-3.3-1.8)\end{array}$ & .397 & $\begin{array}{c}-.457 \\
(-1.5-0.6)\end{array}$ & 0.077 & $\begin{array}{c}.98 \\
(-0.1-2)\end{array}$ \\
\hline$\geq 4$ comorbidities & $0.031^{*}$ & $\begin{array}{c}3.4 \\
(0.3-6 .)\end{array}$ & $0.031^{*}$ & $\begin{array}{c}2.8 \\
(0.2-5.3)\end{array}$ & .512 & $\begin{array}{c}.344 \\
(-0.6-1.3)\end{array}$ & 0.604 & $\begin{array}{c}.28 \\
(-0.7-1.3)\end{array}$ \\
\hline R square & & 0.156 & & & & .076 & & 0.104 \\
\hline
\end{tabular}

Multiple linear regression is statistically significant at the $\mathrm{P}$-value $<0.05$ level

Reference category

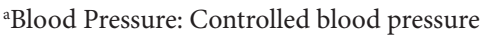

bDuration of hypertension: 1- 5 years

cPolypharmacy: No polypharmacy

${ }^{\mathrm{d}}$ No. of other comorbidity: 1 comorbidity

According to a systematic study conducted by Shahin et al., social support such as disease awareness and reminders from family members and friends have a beneficial effect on medication adherence among hypertension patients. $^{18}$ This study demonstrates a considerable improvement in medication adherence among hypertensive patients with more children, owing to children's additional social and emotional support. ${ }^{19}$ On the contrary, family members have little effect or no effect on Chinese patients' drug adherence. ${ }^{20}$ Other socio-demographic variables reported in this study do not appear to substantially affect patient medication adherence. Similarly, Biffi et al. found no evidence of a significant influence of sex on medication adherence in hypertensive patients; nevertheless, research conducted among Indian hypertensive patients discovered considerable impacts. ${ }^{6,21}$
A study conducted among Korean patients discovered that sex, marital status, smoking, and education did not affect medication adherence, which was consistent with the findings of our study. ${ }^{22}$ In contrast, age has a substantial effect on medication adherence among Korean patients, which contradicts the results of this study.2 In addition, age and education both affect medication adherence in Chinese hypertension patients, which contradicts the findings of this study. ${ }^{20}$ Both studies, however, conclude that gender and marital status did not affect medication adherence. ${ }^{20}$ The review of these studies reveals that most studies impact age on medication adherence; maybe due to the small sample size, this study couldn't find its impact.

This study shows a considerable decline in the adherence to antihypertensive drugs with an increase in comorbidities. In addition, 
the survey conducted in Ethiopia and Egypt also found a significant negative impact of comorbidities in medication adherence. ${ }^{23,24}$ Therefore, the possible reason may be that patients with no comorbidities have simple medication regimens, which help better compliance. On the other hand, the systemic review conducted by Gast and mathes found an uncertain impact of physical comorbidities on the medication adherence of patients suffering from cardiovascular comorbidities. ${ }^{25}$

The duration of hypertension is one of the critical factors which impacts medication adherence among the patients. This study found that medication adherence is reduced in patients suffering from hypertension from 6 to 10 years. Similarly, the study conducted in China also showed poor medication adherence among patients suffering from hypertension from 5 to 15 years; however, Pakistan's study showed no effect. ${ }^{26,27}$ The probable reason is that patients are initially more afraid of their disorder, causing them to be more adherent. Patients gradually develop carelessness and a lack of anxiety about hypertension as time passes.

\section{Study Limitation}

Firstly, the sample size of this study was small, so many factors which impact medication adherence can't be determined. Secondly, some patients provided socially acceptable answers, thus not revealing the truth. Furthermore, this study followed a cross-sectional study design, so cause and effect can't be determined. Finally, the study didn't use direct methods like blood serum or urine analysis to detect medication adherence or indirect methods like the pill count method to precisely determine medication adherence.

\section{CONCLUSION}

This study concludes that three by fourth of the participants are adherent to their antihypertensive medication. Furthermore, the patient's medication adherence is improved when they have more children. On the contrary, their adherence is dropped when they have more comorbidities and suffer from hypertension from 6 to 10 years.

\section{ACKNOWLEDGEMENT}

We are thankful to Dr Shailesh Yadav, Umme Ayman, and Jamuna TR for their invaluable contribution to data collection and ethical clearance.

\section{CONFLICT OF INTEREST}

The authors declare no conflict of interest.

\section{REFERENCES}

1. Singh S, Shankar R, Singh GP. Prevalence and associated risk factors of hypertension: A cross-sectional study in urban Varanasi. Int $\mathrm{J}$ Hypertens. 2017:2017:5491838. doi: 10.1155/2017/5491838, PMID 29348933.

2. Chia YC, Devaraj NK, Ching SM, Ooi PB, Chew MT, Chew BN, et al. Relationship of an adherence score with blood pressure control status among patients with hypertension and their determinants: Findings from a nationwide blood pressure screening program. J Clin Hypertens (Greenwich). 2021;23(3):638-45 doi: 10.1111/jch.14212, PMID 33586334.

3. Ramakrishnan S, Zachariah G, Gupta K, Shivkumar Rao JS, Mohanan PP, Venugopal K, et al. adults: Results from the great India blood pressure survey. Indian Heart J. 2019;71(4):309-13. doi: 10.1016/j.ihj.2019.09.012, PMID 31779858.

4. Available from: apps.who.int. [Adherence to long-term therapies]. World Health Organization; updated 2003; [cited Jun 20 2021]. Available from: http://apps. who.int/iris/bitstream/handle/10665/42682/9241545992.pdf?sequence=1.

5. Sudharsanan N, Ali MK, McConnell M. Hypertension knowledge and treatment initiation, adherence, and discontinuation among adults in Chennai, India: A cross-sectional study. BMJ Open. 2021;11(1):e040252. doi: 10.1136/bmjopen2020-040252, PMID 33472779.
6. Mutneja E, Yadav R, Dey AB, Gupta P. Frequency and predictors of compliance among patients taking antihypertensive medicines. Indian Heart J. 2020;72(2):136-9. doi: 10.1016/j.ihj.2020.03.008, PMID 32534690.

7. [STROBE checklist]. Strobe. updated 2007.

8. Jager J, Putnick DL, Bornstein MH. II. II. More than just convenient: The scientific merits of homogeneous convenience samples. Monogr Soc Res Child Dev. 2017:82(2):13-30. doi: 10.1111/mono.12296, PMID 28475254

9. Kim MT, Hill MN, Bone LR, Levine DM. Development and testing of the hill-bone compliance to high blood pressure therapy scale. Prog Cardiovasc Nurs. 2000;15(3):90-6. doi: 10.1111/j.1751-7117.2000.tb00211.x, PMID 10951950.

10. Kim EY, Han HR, Jeong S, Kim KB, Park H, Kang E, et al. Does knowledge matter?: intentional medication nonadherence among middle-aged Korean Americans with high blood pressure. J Cardiovasc Nurs. 2007:22(5):397-404. doi: 10.1097/01.JCN.0000287038.23186.bd, PMID 17724422.

11. Lambert EV, Steyn K, Stender S, Everage N, Fourie JM, Hill M. Cross-cultural validation of the hill-bone compliance to high blood pressure therapy scale in a South African, primary healthcare setting. Ethn Dis. 2006;16(1):286-91. PMID 16599385.

12. Nashilongo MM, Singu B, Kalemeera F, Mubita M, Naikaku E, Baker A, et al. Assessing adherence to antihypertensive therapy in primary health care in Namibia: Findings and implications. Cardiovasc Drugs Ther. 2017;31(5-6):565-78. doi: 10.1007/s10557-017-6756-8, PMID 29032396.

13. Masnoon N, Shakib S, Kalisch-Ellett L, Caughey GE. What is polypharmacy? A systematic review of definitions. BMC Geriatr. 2017;17(1):230. doi: 10.1186/ s12877-017-0621-2, PMID 29017448.

14. Available from: emro.who.int. [High blood pressure: a public health problem] World Health Organization. Available from: http://www.emro.who.int/media/ world-health-day/public-health-problem-factsheet-2013.html [cited 25/10/2021]

15. Available from: wma.net [World Medical Association] WMA Declaration of Helsinki - Ethical Principles for Medical Research Involving Human Subjects - WMA - The World Medical Association [cited Jul 10 2021]. Available from: https://www.wma.net.

16. Uchmanowicz B, Chudiak A, Uchmanowicz I, Rosinczuk J, Froelicher ES Factors influencing adherence to treatment in older adults with hypertension. Clin Interv Aging. 2018;13:2425-41. doi: 10.2147/CIA.S182881, PMID 30568434.

17. Abu-El-Noor NI, Aljeesh YI, Bottcher B, Abu-El-Noor MK. Assessing barriers to and level of adherence to hypertension therapy among palestinians living in the Gaza Strip: A chance for policy innovation. Int J Hypertens. 2020;2020:7650915. doi: 10.1155/2020/7650915, PMID 33062318.

18. Shahin W, Kennedy GA, Stupans I. The association between social support and medication adherence in patients with hypertension: A systematic review. Pharm Pract (Granada). 2021;19(2):2300. doi: 10.18549/PharmPract. 2021.2.2300, PMID 34221197.

19. Thomas PA, Liu H, Umberson D. Family relationships and well-being. Innov Aging. 2017;1 (3):igx025. doi: 10.1093/geroni/igx025, PMID 29795792.

20. Zhang $Y$, Li X, Mao L, Zhang M, Li K, Zheng $Y$, et al. Factors affecting medication adherence in community-managed patients with hypertension based on the principal component analysis: Evidence from Xinjiang, China. Patient Prefe Adherence. 2018;12:803-12. doi: 10.2147/PPA.S158662, PMID 29785095.

21. Biffi A, Rea F, lannaccone T, Filippelli A, Mancia G, Corrao G. Sex differences in the adherence of antihypertensive drugs: A systematic review with metaanalyses. BMJ Open. 2020;10(7):e036418. doi: 10.1136/bmjopen-2019-036418, PMID 32641331.

22. Choi HY, Oh IJ, Lee JA, Lim J, Kim YS, Jeon TH, et al. Factors affecting adherence to antihypertensive medication. Korean J Fam Med. 2018;39(6):325-32. doi: 10.4082/kjfm.17.0041, PMID 30384549.

23. Hussein A, Awad MS, Mahmoud HEM. Patient adherence to antihypertensive medications in upper Egypt: A cross-sectional study. Egypt Heart J. 2020;72(1):29. doi: 10.1186/s43044-020-00066-0, PMID 32451726.

24. Shokoohizadeh L, Ekrami A, Labibzadeh M, Ali L, Alavi SM. Antimicrobial resistance patterns and virulence factors of enterococci isolates in hospitalized burn patients. BMC Res Notes. 2018:11(1):1. doi: 10.1186/s13104-017-3088-5. PMID 29291749.

25. Gast A, Mathes T. Medication adherence influencing factors-an (updated) overview of systematic reviews. Syst Rev. 2019;8(1):112. doi: 10.1186/s13643-0191014-8, PMID 31077247

26. Pan J, Wu L, Wang H, Lei T, Hu B, Xue $X$, et al. Determinants of hypertension treatment adherence among a Chinese population using the therapeutic adherence scale for hypertensive patients. Med (Baltim). 2019;98(27):e16116 doi: 10.1097/MD.0000000000016116, PMID 31277112

27. Boratas S, Kilic HF. Evaluation of medication adherence in hypertensive patients and influential factors. Pak J Med Sci. 2018;34(4):959-63. doi: 10.12669/ pjms.344.14994, PMID 30190761. 